\title{
AVALIAÇÃO DO CONHECIMENTO SOBRE OS NOVOS EPIS DURANTE A PANDEMIA NO SERVIÇO PÚBLICO DO MUNICÍPIO DE CAJAZEIRAS-PB: UM ESTUDO COM CIRURGIÕES-DENTISTAS
}

\author{
ASSESSMENT OF KNOWLEDGE ABOUT THE NEW PPE DURING \\ THE PANDEMIC IN THE PUBLIC SERVICE OF THE MUNICIPALITY \\ OF CAJAZEIRAS-PB: A STUDY WITH DENTAL SURGEONS
}

Gilmara Araújo de Sousa ${ }^{1}$ Kyara Dayse de Souza Pires ${ }^{2}$

Rodolfo de Abreu Carolino ${ }^{3}$

Lívia Pereira Brocos Pires ${ }^{4}$

Lilliane Juvenal Andrade ${ }^{5}$

Rafaela da Costa Holanda 6

José Klidenberg de Oliveira Júnior ${ }^{7}$

RESUMO: Introdução: Os cirurgiões-dentistas (CD) constituem um grupo de risco devido ao alto contato com fluidos e aerossóis durante o atendimento odontológico. Torna-se relevante a realização de estudos que observem se estes profissionais estão cumprindo as medidas de biossegurança com o uso de dispositivos apropriados, bem como se estão utilizando os novos equipamentos de proteção individual (EPI) acrescentados durante a pandemia. Objetivo: Avaliar 0 conhecimento dos cirurgiões-dentistas inseridos no serviço público do município de Cajazeiras-PB, sobre o uso e a importância dos novos dispositivos de proteção

\footnotetext{
Graduada em Odontologia pela Faculdade Santa Maria de Cajazeiras - PB. gilmaratsb@outlook.com.

2 Graduada em Odontologia pela Universidade Federal de Campina Grande. kyaraodonto@gmail.com.br.

${ }^{3}$ Mestre em Odontologia pela Universidade Estadual da Paraíba, docente do curso de Odontologia da Faculdade Santa Maria de Cajazeiras-PB. rodolfoorg@yahoo.com.br.

${ }^{4}$ Mestre em Odontologia pela Universidade Potiguar, docente do curso de Odontologia da Faculdade Santa Maria de Cajazeiras. liviabrocos@gmail.com.

5 Graduada em Odontologia pela Faculdade Santa Maria de Cajazeiras - PB. Lillianejuvenal123@hotmail.com.

${ }^{6}$ Mestre em Odontologia pela Faculdade de Odontologia de Piracicaba/FOP-UNICAMP, docente do curso de Odontologia da Faculdade Santa Maria de Cajazeiras-PB. rafaela_ico@hotmai.com.

${ }^{7}$ Mestre em Odontologia pela Universidade Federal da Paraíba, docente do curso de Odontologia da Faculdade Santa Maria de Cajazeiras-PB. joseklidemberg@gmail.com.
} 
respiratória durante a pandemia. Metodologia: $O$ estudo refere-se a um estudo transversal realizado com 25 cirurgiões-dentistas da cidade de Cajazeiras-PB. Foram coletados os dados sociodemográficos e referentes ao entendendo sobre biossegurança e novos EPIs acrescidos através de questionário previamente estruturado pelos pesquisadores. Foi empregada a estatística descritiva, por meio de frequência absoluta e relativa dos dados, para caracterização da amostra. Resultados: Dos 25 participantes, 64\% $(n=16)$ eram do sexo feminino. Todos os entrevistados relataram ter ciência dos riscos biológicos da profissão, entretanto só $62,5 \%(n=15)$ disseram receber equipamentos suficientes de proteção nas Unidades Básicas de Saúde (UBS). A COVID -19 é uma doença que causa temor nos dentistas, visto que $92 \%(n=23)$ confirmaram sentir medo em adquiri-la durante 0 atendimento. Entre os equipamentos mais eficazes na proteção, 88\% $(n=22)$ citaram fazer uso dos respiradores N95, com e sem válvula e $80 \%(n=22)$ dos cirurgiõesdentistas citaram usar o protetor facial. Por fim, $88 \%(n=22)$ responderam que utilizavam o respirador N95 sem válvula e $28 \%(n=7)$ disseram que o reutilizam por 15 dias. Conclusão: Conclui-se que a maioria dos cirurgiões-dentistas fazia uso desses equipamentos de proteção individual durante o atendimento odontológico assistencial com indicações corretas de seu uso.

PALAVRAS CHAVE: Conhecimento, Covid-19, Equipamento de proteção individual, Odontologia.

ABSTRACT: Introduction: Dental surgeons (DC) constitute a risk group due to high contact with fluids and aerosols during dental care. It is relevant to conduct studies that observe whether these professionals are complying with biosecurity measures with the use of appropriate devices, as well as whether they are using the new personal protective equipment (PPE) added during the pandemic. Objective: Evaluating the knowledge of dentists inserted in the public service of the city of Cajazeiras-PB, about the use and importance of new respiratory protection devices during the pandemic. Methodology: The study refers to a cross-sectional study conducted with 25 dentists in the city of Cajazeiras-PB. Sociodemographic data and data referring to the understanding of biosafety and new PPE added through a questionnaire previously structured by the researchers were collected. Descriptive statistics through absolute and relative frequency of data were used to characterize the sample. Results: Of the 25 participants, 64\% $(N=16)$ were female. All respondents reported being aware of the biological risks of the profession, however only $62.5 \%(\mathrm{~N}=15)$ said they received sufficient protective equipment in Basic Health Units (UBS). COVID -19 is a disease that causes fear in dentists, as $92 \%(\mathrm{~N}=23)$ confirmed being afraid to acquire it during the service. Among the most effective protective equipment, 88\% (N=22) mentioned using $N 95$ respirators, with and without valves, and $80 \%(\mathrm{~N}=22)$ of dentists mentioned using face shields. Finally, $88 \%$ $(N=22)$ responded that they used the $N 95$ respirator without a valve and $28 \%(N=7)$ said that they reused it for 15 days. Conclusion: It is concluded that most dentists used these personal protective equipment during dental care with correct indications for its use.

KEYWORDS: Knowledge, Covid-19, Individual protection equipment, Dentistry. 


\section{INTRODUÇÃO}

O consultório odontológico é um ambiente que possui uma grande carga de agentes patogênicos. Dessa forma, a odontologia é considerada uma profissão que traz um maior risco de contaminação. Entre os demais riscos biológicos aos quais os cirurgiões-dentistas (CDs) e seus pacientes estão expostos, está a infecção cruzada por gotículas infectadas e aerossóis dentro do ambiente clínico. Essa infecção pode ocorrer de paciente para profissional, profissional para paciente e profissional para equipe (NOGUEIRA; BASTOS, COSTA, 2010; ARANTES et al., 2015).

O cenário atual de pandemia pela COVID -19 protagonizado pelo novo corona vírus, SARS-CoV-2, trouxe para a odontologia uma mudança no protocolo de atendimento e novos dispositivos de proteção individual (ARANTES et al, 2015; MONTALLI et al., 2020). Durante os procedimentos odontológicos, como por exemplo, o uso da caneta de alta rotação e seringa tríplice é lançada uma grande carga patogênica dentro do consultório o que deixa o ambiente contaminado, trazendo um risco extensivo aos demais membros da equipe e público assistido (PERES; BOLÉO-TOME; SANTOS, 2020).

Diante a essa realidade, vários países e suas associações odontológicas recomendaram ou mesmo compeliram os dentistas a adiar procedimentos eletivos e suas atividades profissionais foram restritas apenas a emergências. Foi necessário acrescentar o uso de novos dispositivos de proteção como os respiradores N95, PFF2, face shield para a equipe de saúde bucal. Estes equipamentos possuem uma alta capacidade de prevenir a passagem de fluídos orgânicos quando usados de forma adequada (FRANCO; CAMARGO; PERES, 2020).

Além do mais é indispensável que o CD tenha um conhecimento apropriado sobre esses dispositivos que foram incluídos na paramentação odontológica, devendo saber como e quando utilizá-los, duração do tempo de eficiência e o manejo quando for fazer a reutilização desses dispositivos de proteção respiratória (BARBIERI et al., 2019). 
Por isso, torna-se importante investigar a utilização dos equipamentos de proteção individual por parte dos profissionais de saúde, em especial, os cirurgiõesdentistas já que constituem um grupo de risco devido ao alto contato com fluidos e aerossóis durante os atendimentos odontológicos. Observando se estão cumprindo as medidas de biossegurança da melhor forma possível, como também, se fazem parte do cotidiano destes o uso dos dispositivos já utilizados e os novos que foram acrescentados como os protetores respiratório N95, N99, PFF2, PFF3 e o face shield (FRANCO; CAMARGO; PERES, 2020).

Vale ressaltar que a biossegurança deve estar sempre presente em mente e sendo praticada da melhor forma pelos profissionais para ter uma maior prevenção e não contrair as infecções (MADUREIRA, 2015).

Mediante a este contexto, esta pesquisa teve como objetivo avaliar o conhecimento dos cirurgiões-dentistas inseridos no serviço público do município de Cajazeiras-PB, sobre o uso e a importância dos novos dispositivos de proteção respiratória durante a pandemia.

\section{METODOLOGIA}

A pesquisa obedeceu a todas as recomendações provenientes da Resolução 466/12 do Conselho Nacional de Saúde (CNS) referente a estudos envolvendo seres humanos, considerando a privacidade e os direitos dos participantes. $\mathrm{O}$ número do parecer de aprovação do comitê de ética em pesquisa foi 4.581.896.

Trata-se de um estudo transversal realizado em 2021 com uma amostra de CDs que trabalhavam nas UBS e no Centro de Especialidades Odontológicas do município de Cajazeiras-PB. Foi adotada uma amostra por conveniência ajustado para populações finitas de CDs atuantes nas UBS. Para o cálculo amostral, foram adotados a prevalência de conhecimento do CDs de $50 \%$, poder do teste de $80 \%$, intervalo de confiança de $95 \%$ e estimativa de erro de $5 \%$. A partir desse cálculo seria necessário 384 CDs. Foi realizado o ajuste para populações finitas, totalizando 
26 cirurgiões-dentistas para a pesquisa. Para a realização do teste foi utilizado o Epilnfo program (versão 7).

Critérios de elegibilidade

Foram incluídos no estudo cirurgiões-dentistas que trabalhavam na rede assistencial de saúde pública de Cajazeiras-PB, de ambos os sexos, que estavam presentes na Unidade no momento de realização da pesquisa. Foram excluídos profissionais que tinham menos de um mês de atuação da rede pública.

Coleta de Dados

Os dados foram coletados através de um questionário pré-estruturado elaborado pelo pesquisador. Cada CD recebeu o instrumento de pesquisa via Google Forms, acompanhado com o Termo de Consentimento Livre e Esclarecido (TCLE). O questionário continha 12 perguntas relacionadas ao conhecimento que esses profissionais tinham sobre o uso de Equipamentos de Proteção Individual em seu ambiente de trabalho. Além do mais, continham informações sobre as características sociodemográficas dos participantes da pesquisa como: sexo, idade, tempo de formação e a disponibilização dos EPIs na unidade de saúde.

\section{Estudo Piloto}

Realizou-se o estudo piloto envolvendo 10 profissionais da área que não participaram do estudo principal. Através desse estudo, a metodologia foi testada. Os resultados do estudo piloto indicaram que mudanças na metodologia proposta 
não eram necessárias. Além disso, previamente ao estudo piloto, foi realizado o teste-reteste do questionário para verificar o entendimento das perguntas elaboradas pelos pesquisadores. Este teste foi administrado aos cirurgiões-dentistas por duas vezes tendo um intervalo de 15 dias entre a primeira aplicação e a última.

Análise Estatística

A análise estatística ocorreu através do programa software Statistical Package for Social Science (Versão 22.0, IBM SPPS Inc., Armonk, NY, USA). Empregou-se a estatística descritiva, por meio de frequência absoluta e relativa, para caracterização da amostra e distribuição das variáveis do estudo.

\section{RESULTADOS E DISCUSSÃO}

A pesquisa realizada teve uma amostra de 25 participantes do universo da amostragem que é aproximadamente de $30 \mathrm{CDs}$ do serviço público do município de Cajazeiras-PB. Entre estes, 64\% $(n=16)$ eram do sexo feminino, 80\% $(n=20)$ possuíam uma pós-graduação, 64\% ( $n=16)$ fizeram uma especialização e $72 \%$ $(n=18)$ atuavam tanto no setor privado como público. Em relação ao tempo de formação, $16 \%(n=4)$ afirmaram ter se formado há 2 anos, sendo o maior percentual entre as respostas obtidas.

Esses dados obtidos também foram evidenciados em outros estudos similares (MORAIS et al., 2020; SOUZA; SILVA; LOUREIRO; ZUZA, 2021). Quanto aos profissionais trabalharem em ambos os setores público e privado, notou-se que após a criação de políticas públicas de saúde voltadas a atenção para a saúde bucal, mais de um terço dos profissionais de odontologia do Brasil estavam atuando dentro do Sistema Público de Saúde (CASCAES; DOTTO; BOMFIM, 2018). No entanto, muitos profissionais atuam em conjunto com o setor privado. 
Indagados quais doenças respiratória causavam algum temor durante o atendimento odontológico, 92\% $(\mathrm{n}=23)$ responderam "SIM" para o Corona vírus, conforme exposto na Tabela 1. O resultado encontrado se mostrou em concordância com a realidade vivenciada, visto que a pandemia da Covid-19 gerou grande preocupação devido ao seu alto índice de transmissão e infecção. Este contexto causou temor nos profissionais que trabalhavam diariamente com esse risco (SOUZA; SILVA; LOUREIRO; ZUZA, 2021).

Tabela 1 - Doenças respiratórias que causam algum temor.

\begin{tabular}{lccc}
\hline Doenças & & Frequência & Percentual \\
\hline \multirow{2}{*}{ H1N1 tipo A } & Sim & 18 & 72,0 \\
\cline { 2 - 4 } & Não & 7 & 28,0 \\
\cline { 2 - 4 } H1N1 tipo B & Sim & 14 & 56,0 \\
\cline { 2 - 4 } Gripe Espanhola & Não & 11 & 44,0 \\
\cline { 2 - 4 } Gripe & Sim & 4 & 16,0 \\
\cline { 2 - 4 } Corona vírus & Não & 21 & 84,0 \\
\cline { 2 - 4 } & Sim & 11 & 44,0 \\
\hline \multirow{2}{*}{ Hantavirose } & Não & 14 & 56,0 \\
\hline \multirow{2}{*}{ Tuberculose } & Sim & 23 & 92,0 \\
\cline { 2 - 4 } & Não & 2 & 8,0 \\
\hline
\end{tabular}

Fonte: Do autor (2021).

A Tabela 2 apresentou as respostas das perguntas feitas aos profissionais sobre capacitação de biossegurança durante a pandemia. Verificou-se que apenas $24 \%(n=6)$ receberam essa capacitação e $88 \%(n=22)$ dos participantes relataram terem interesse e sentem necessidade em recebê-la. Esta situação foi constatada também na pesquisa de Gaspar et al. (2020), onde mais de um terço de sua amostra não teve nenhum tipo de treinamento ou curso de biossegurança no período da pandemia, o que pode acarretar o mau uso dos equipamentos de proteção e um alto risco de contaminação. 
Tabela 2- Capacitação sobre biossegurança durante a pandemia.

\begin{tabular}{|c|c|c|c|}
\hline Pergunta & & Frequência & Percentual \\
\hline $\begin{array}{l}\text { Recebeu capacitação de biossegurança } \\
\text { na pandemia }\end{array}$ & $\begin{array}{l}\text { Sim } \\
\text { Não }\end{array}$ & $\begin{array}{c}6 \\
19\end{array}$ & $\begin{array}{l}24,0 \% \\
76,0 \%\end{array}$ \\
\hline $\begin{array}{l}\text { Gostaria de receber capacitação de } \\
\text { biossegurança na pandemia }\end{array}$ & $\begin{array}{l}\text { Sim } \\
\text { Não }\end{array}$ & $\begin{array}{c}22 \\
3\end{array}$ & $\begin{array}{l}88,0 \% \\
12,0 \%\end{array}$ \\
\hline
\end{tabular}

Fonte: Do autor (2021).

Todos os participantes da pesquisa afirmaram terem ciência dos riscos biológicos que as doenças respiratórias podem acometer ao profissional da odontologia. Outro fato evidenciado foi que $62 \%(n=15)$ destes, afirmaram ter os equipamentos adequados à sua disposição com o número suficiente para os atendimentos (Gráfico 1).

Gráfico 1 - Disponibilização dos equipamentos adequados pela Unidade Básica de Saúde em número suficiente para atendimento.

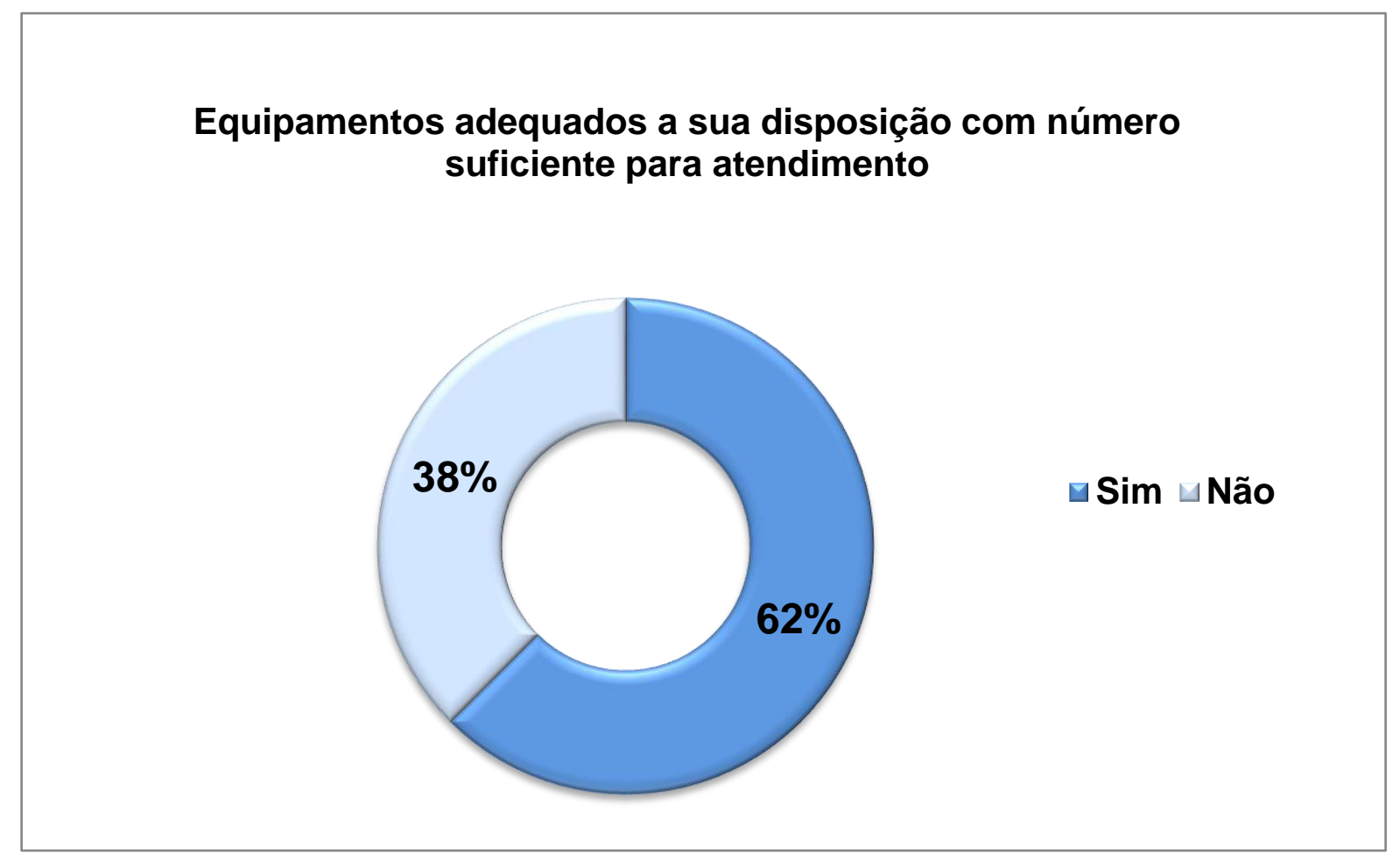

Fonte: Dados da Pesquisa. 
Os protocolos da pandemia da COVID-19 dispõem sobre a troca dos EPIs por atendimento essencial (FRANCO; CAMARGO; PERES, 2020). Esse dado obtido na pesquisa não é de acordo com o que é indicado pelos protocolos sugeridos pela Agência Nacional de Vigilância Sanitária (ANVISA, 2020) e do Conselho Federal de Odontologia (CFO, 2020).

Segundo estes, cada profissional tem que utilizar no mínimo 10 respiradores por semana, fazendo o uso de um respirador por turno de atendimento e logo, em seguida, sendo removido e guardado no recipiente plástico com tampa perfurada e dando um intervalo de no mínimo cinco dias para reutilizar novamente. A cada uso, identificar a quantidade de vezes que foi utilizado, não podendo ultrapassar mais de quinze vezes de uso (FRANCO; CAMARGO; PERES, 2020).

Questionados sobre quais EPIs os participantes da pesquisa usavam em seus atendimentos, os que apresentaram maiores porcentagens foram: N95 sem válvula (88\% $n=22)$, protetor facial $(88 \% n=22)$, óculos de proteção $(64 \% n=16)$, avental $(88 \% n=22)$ e luvas $(96 \% n=24)$ (Tabela 3). Esses achados estão de acordo com os protocolos propostos pela Associação de Medicina Intensiva Brasileira (AMIB, 2020) e CFO (2020) em relação ao manejo clínico e enfrentamento diante da pandemia COVID-19. Constatou-se que a grande maioria dos profissionais estão atentos às normas técnicas publicadas pelos órgãos responsáveis. 
Tabela 3 - Equipamentos de proteção individual que você utiliza no atendimento.

\begin{tabular}{|c|c|c|c|}
\hline $\begin{array}{l}\text { Equipamentos de proteção individual } \\
\text { que você utiliza no atendimento }\end{array}$ & & Frequência & Percentual \\
\hline Máscara cirúrgica & $\begin{array}{l}\text { Sim } \\
\text { Não }\end{array}$ & $\begin{array}{l}11 \\
14\end{array}$ & $\begin{array}{l}44 \% \\
56 \%\end{array}$ \\
\hline \multirow{2}{*}{ N99 sem válvula } & Sim & 24 & $96 \%$ \\
\hline & Não & 1 & $4 \%$ \\
\hline \multirow{2}{*}{ N99 com válvula } & Sim & 0 & 0 \\
\hline & Não & 25 & $100 \%$ \\
\hline N95 sem válvula & $\operatorname{Sim}$ & 22 & 88 \\
\hline \multirow[b]{2}{*}{ N95 com válvula } & Não & $\begin{array}{l}3 \\
1\end{array}$ & 12 \\
\hline & Não & 24 & $\begin{array}{c}4 \\
96\end{array}$ \\
\hline \multirow{2}{*}{ PFF/2 } & Sim & 7 & 28 \\
\hline & Não & 18 & 72 \\
\hline \multirow{2}{*}{ PFF/3 } & Sim & 1 & 4 \\
\hline & Não & 24 & 96 \\
\hline \multirow{2}{*}{ Máscara de tecido } & Sim & 0 & 0 \\
\hline & Não & 25 & 100 \\
\hline \multirow{2}{*}{ Protetor facial } & Sim & 22 & 88 \\
\hline & Não & 3 & 12 \\
\hline \multirow{2}{*}{ Óculos de proteção } & Sim & 16 & 64 \\
\hline & Não & 9 & 36 \\
\hline \multirow{2}{*}{ Gorro } & Sim & 24 & 96 \\
\hline & Não & 1 & 4 \\
\hline \multirow{2}{*}{ Avental } & Sim & 22 & 88 \\
\hline & Não & 3 & 12 \\
\hline \multirow{2}{*}{ Jaleco } & Sim & 10 & 40 \\
\hline & Não & 15 & 60 \\
\hline \multirow{2}{*}{ Luvas } & Sim & 24 & 96 \\
\hline & Não & 1 & 4 \\
\hline \multirow{2}{*}{ Pro-pé } & Sim & 5 & 20 \\
\hline & Não & 20 & 80 \\
\hline
\end{tabular}

Fonte: Do Autor (2021).

Contudo, é preciso que os profissionais também possam fazer o uso frequente de todos os materiais propostos pelos protocolos, como o pro-pé e o jaleco, para que as medidas de proteção possam ser eficientes e evitem a contaminação (HERRERA-PLASENCIA; ENOKI-MIÑANO; RUIZ-BARRUETO, 2020). A literatura relatou que, o grande número de acidentes relacionados a exposição infecciosa de agentes biológicos está relacionado ao mau uso ou uso incorreto dos EPIs (ARAÚJO \& COSTA, 2016). 
O ideal é que os respiradores sejam sem válvula de exalação, pois, ao serem utilizados, eles contendo válvula podem exalar agentes patogênicos no ambiente e assim colocando em risco de contaminação as pessoas presentes no ambiente, ficando recomendado somente o uso sem a válvula de exalação (SOARES et al., 2020).

Outro dado interessante da pesquisa diz respeito ao tempo de reutilização e forma de armazenamento do respirador N95 pelos CDs (Tabela 4). Quando perguntado sobre por quanto tempo eles o reutilizavam, $28 \%(n=7)$ responderam que reutilizavam durante 15 dias. Os que responderam que reutilizavam o dispositivo durante 7, 5 e 3 dias apareceram com frequência relativa de 12\% $(n=3)$. Sobre como guardavam o respirador, $28 \%(n=7)$ afirmaram que utilizavam o grau cirúrgico, $20 \%$ $(n=5)$ que utilizavam um recipiente plástico e $12 \%(n=3)$ que faziam o uso de papel toalha.

Tabela 4 - Tempo de utilização do respirador N95 e forma como o guarda.

\begin{tabular}{lccc}
\hline & & Frequência & Percentual \\
\hline & 15 dias & 7 & 28,0 \\
& 7 dias & 3 & 12,0 \\
Por quanto tempo você & 5 dias & 3 & 12,0 \\
reutiliza seu respirador & 3 dias & 3 & 12,0 \\
N95? & 10 dias & 2 & 8,0 \\
& 2 dias & 2 & 8,0 \\
& 1 dia & 1 & 4,0 \\
& 14 dias & 1 & 4,0 \\
& Sempre & 1 & 4,0 \\
& Nunca & 2 & 8,0 \\
Como você guarda & Grau cirúrgico & 7 & 28,0 \\
respirador N95? & Na embalagem & 3 & 12,0 \\
& Recipiente plástico & 5 & 20,0 \\
& Envelope & 2 & 8,0 \\
& Em papel toalha & 3 & 12,0 \\
& Sacola descartável & 2 & 8,0 \\
& Bolsinha de & 1 & 4,0 \\
& máscara & 2 & 8,0 \\
\hline
\end{tabular}

Fonte: Do autor (2021). 
Silva et al. (2021) apresentaram que o ideal seria o descarte do equipamento logo após o atendimento, mas em necessidade de reuso não deveria se ultrapassar de cinco dias. Contudo a pesquisa realizada mostra que $52 \%(n=15)$ da amostra reutiliza seu respirador por mais tempo que o indicado. Este fato pode ter-se dado tanto pela escassez de equipamentos adequados devido à alta demanda durante a pandemia ao redor do mundo, o que ocasionou a falta de equipamentos para os profissionais do setor de saúde (Silveira et al., 2021).

Como também pelo aumento exorbitante do preço de tais equipamentos e a falta de conhecimento dos profissionais sobre o uso dos novos EPIs, dificultando a troca adequada do respirador de acordo com as demandas do CD (SOUZA; SILVA; LOUREIRO; ZUZA, 2021; SILVA; TOMAZ; BARJA; VIRIATO, 2021).

Ademais, para que possa ser realizada essa reutilização, os respiradores N95/PFF2 devem ser armazenados de preferência em recipientes descartáveis, como em papel toalha ou folha de papel A4. E pode ser armazenado em recipiente plástico perfurado (MATTE; CACAU; REIS; ASSIS, 2020), o que vai ao encontro do resultado desta pesquisa, onde $56 \%(n=14)$ da amostra relataram utilizar os materiais descartáveis para guardar o equipamento.

Outro tópico importante se refere a como o profissional $C D$ faz a paramentação e a desparamentação do seu equipamento após o atendimento, pois a sequência realizada de forma correta pode auxiliar na diminuição do risco de contagio. Podemos observar na Tabela 5 que, $80 \%(n=20)$ da amostra selecionou a sequência 1 (higiene das mãos, jaleco, avental, pro-pé, higienizar as mãos, respirador, verificar máscara, teste de vedação, óculos de proteção, gorro, protetor facial e luva) como a que utilizou para a paramentação do EPI e 52\% ( $n=13)$ afirmou que faz a sequência 1 (luvas, avental, protetor facial, óculos, gorro, jaleco, respirador, higienizar mãos, máscara nova e álcool nas mãos) para desparamentação. 
Tabela 5 - Resultados referentes a paramentação e desparamentação do equipamento.

\begin{tabular}{|c|c|c|c|}
\hline $\begin{array}{lr}\text { Paramentação } & \text { e } \\
\text { Desparamentação } & \text { do } \\
\text { equipamento } & \\
\end{array}$ & & Frequência & Percentual \\
\hline \multirow{5}{*}{$\begin{array}{l}\text { Qual sequência você faz a } \\
\text { paramentação } \\
\text { equipamentos de } \\
\text { individual? } \\
\text { indoteção } \\
\text { Qual sequência você faz a } \\
\text { desparamentação } \\
\text { equipamentos de proteção facial } \\
\text { e respiratória? }\end{array}$} & $\begin{array}{l}\text { Sequência } 1 \\
\text { Sequência } 2\end{array}$ & $\begin{array}{c}20 \\
4\end{array}$ & $\begin{array}{l}80,0 \\
16,0\end{array}$ \\
\hline & Não respondido & 1 & 4,0 \\
\hline & Sequência 1 & 13 & 52,0 \\
\hline & Sequência 2 & 8 & 32,0 \\
\hline & Não respondido & 4 & 16,0 \\
\hline $\begin{array}{l}\text { Você faz a paramentação dentro } \\
\text { do consultório? } \\
\text { Você faz a desparamentação } \\
\text { dentro do consultório? }\end{array}$ & $\begin{array}{l}\text { Sim } \\
\text { Não } \\
\text { Sim } \\
\text { Não }\end{array}$ & $\begin{array}{c}25 \\
0 \\
25 \\
0\end{array}$ & $\begin{array}{c}100,0 \\
0,0 \\
100,0 \\
0,0\end{array}$ \\
\hline
\end{tabular}

Fonte: Do autor (2021).

É importante destacar que esses profissionais faziam a paramentação e desparamentação dentro do consultório, não sendo recomendado ser realizada dentro do consultório pelo protocolo. Esses resultados apresentaram que mais da metade da amostra segue de forma correta os protocolos de paramentação e desparamentação dos EPIs, publicada pelos órgãos responsáveis da área da saúde em relação ao atendimento aos pacientes em tempos de pandemia.

Guedes, Cavalcanti e Alencar (2020) disseram ser importante que o CD tenha um conhecimento sobre o correto uso desses dispositivos de proteção respiratória levando em consideração a paramentação e desparamentação dos mesmos. Para a paramentação do profissional, é recomendado seguir o roteiro exposto na figura 1. Se for o primeiro atendimento, pode realizar dentro do consultório. A paramentação entre um paciente e outro deverá ser realizada fora do consultório. Antes de iniciá-la, é preciso fazer a higienização das mãos com água e sabão líquido.

Após colocar o respirador N95 ou PFF2 é necessário adaptá-lo por baixo do queixo com a parte metálica (clip nasal) para cima. Depois ajustar as alças/elásticos na cabeça ou atrás das orelhas, garantindo que não fiquem torcidos. Lembrando que 
o elástico superior deve ficar acima das orelhas e o elástico inferior na nuca. Com ambas as mãos moldar o clip nasal ao redor do nariz e bochechas.

A Organização Mundial de Dentistas sugere que a desparamentação ocorra fora do consultório. Quando o profissional for se desparamentar, deve-se realizar a remoção dos EPls seguindo a ordem demonstrada na figura 2. Luvas, avental descartável, gorro, máscara cirúrgica precisa ser descartada no lixo contaminado. Após o descarte das luvas, higienizar as mãos com água, sabão líquido e secá-las com papel toalha para assim dar continuidade a desparamentação. Ao retirar cada EPI, passar álcool em gel nas mãos, com exceção apenas da N95, visto que depois de retirá-la, o dentista deve lavar as mãos com água e sabão (MELO et al., 2020).

O presente estudo possui algumas limitações que devem ser abordadas. Primeiramente, os resultados foram restritos profissionais do setor público do município de Cajazeiras, o que indica a necessidade de futuros estudos realizados com outras populações do Brasil. Além disso, o estudo transversal não consegue mensurar a relação de causa-efeito entre as variáveis estudadas.

Mesmo a grande maioria possuindo o conhecimento do assunto, uma outra parte dos entrevistados, não sabiam dos protocolos de utilização dos EPIs, seria importante que capacitação sobre educação em biossegurança fossem realizadas pela Coordenação de Saúde Bucal e Secretaria de Saúde do município ou até mesmo pelo Conselho Federal de Odontologia (CFO).

\section{CONCLUSÃO}

Através dessa pesquisa, pôde-se concluir que grande maioria dos CDs que trabalham no serviço público de Cajazeiras possuem significativo conhecimento sobre a biossegurança e a importância do uso dos novos dispositivos de proteção respiratória. N95, PFF2 e o protetor facial (Face shield) foram dispositivos inseridos na rotina clínica odontológica durante o período pandêmico. 
Eles também possuem um conhecimento sobre os riscos biológicos aos quais estão expostos durante $\mathrm{o}$ atendimento assistencial. A doença respiratória mais temida por esses profissionais é a Covid-19.

\section{REFERÊNCIAS BIBLIOGRÁFICAS}

ARANTES, D. C.; HAGES, C. A.; NASCIMENTO, L. A. et al. Biossegurança aplicada à Odontologia na Universidade Federal do Pará, Cidade de Belém, Estado do Pará, Brasil. Rev Pan-Amaz Saúde, Ananindeua, v.6, n.1, p.11-18, 2015.

ARAÚJO, R. L; COSTA, P. A. G. P. A importância do equipamento de proteção individual no âmbito odontológico e uma abordagem sobre 0 atendimento de pacientes com tuberculose [Monografia]. Faculdade São Lucas, Porto Velho, Rondônia, Brasil.dônia, Brasil, 2016.

Agência Nacional de Vigilância Sanitária. (2020). Nota Técnica GVIMS/GGTES/ANVISA oㅡ 04/2020 (revisada em 27/10/2020). https://www.gov.br/anvisa/pt$\mathrm{br} / \mathrm{centraisdeconteudo/publicacoes/servicosdesaude/notas-}$

tecnicas/nota_tecnica_gvims_ggtes_anvisa_04_2020_reviso_27-10-2020.pdf/view.

Associação de Medicina Intensiva Brasileira. (2020). Recomendações AMIB/CFO para atendimento odontológico COVID-19. Comitê de Odontologia AMIB/CFO de enfrentamento ao COVID-19 Departamento de Odontologia AMIB - $3^{\circ}$ Atualização 22/06/2020. São Paulo: Associação de Medicina Intensiva Brasileira. https://www.amib.org.br/fileadmin/user_upload/amib/2020/junho/22/220620Recomendacoes_AMI B_-_CFO_para_atendimento_odontologico_COVID-19.pdf.

BARBIERI, A. A.; FEITOSA, F.; RAMOS, C. J. et al. Biosafety measures in dental practice: Literature Review. Brazilian Dental Science, v.22, n.1., p.9-16, 2019.

CASCAES, A. M.; DOTTO, L.; BOMFIM, R. A. Tendências da força de trabalho de cirurgiõesdentistas no Brasil, no período de 2007 a 2014: Estudo de séries temporais com dados do Cadastro Nacional de Estabelecimentos de Saúde. Epidemiologia e Serviços de Saúde, v.27, n.1, p.1-10, 2018.

Conselho Federal de Odontologia (CFO). Recomendações para atendimentos odontológicos em tempos de covid-19. Brasília, DF: Conselho Federal de Odontologia, 2020. https://website.cfo.org.br/wp-content/uploads/2020/03/Material-CDs-Coronavi\%CC\%81rus-CFO1.pdf.

FRANCO, J. B.; CAMARGO, A. R.; PERES, M. P. S. M. Cuidados Odontológicos na era do COVID-19: recomendações para procedimentos odontológicos e profissionais. Revista da Associação Paulista de Cirurgiões-Dentistas, v.74, n.1, p.18-21, 2020.

GASPAR, G. S.; FIGUEIREDO, N.; LUCENA, E. H. G. et al. Characterization of Dental Surgeons of Pernambuco State in the COVID-19 Pandemic Context: Preliminary Data. Pesquisa Brasileira em Odontopediatria e Clínica Integrada, v.20, n.1, p.1-7, 2020.

GUEDES, R. H. R. G.; CAVALCANTI, T. B. B.; ALENCAR, M. G. M. Protocolo de atendimento de urgência divisão técnica - COVID-19: Higienização dos consultórios após atendimento sem aerossol. Revista Científica OARF, v.4, n.1, p.16-10, 2020. 
HERRERA-PLASENCIA, P. M.; ENOKI-MIÑANO, E.; RUIZ-BARRUETO, M. A. Riesgos, contaminación y prevención frente al COVID-19 en el quehacer odontológico: una revisión. Revista de Salud Pública, v.22, n.5, p.1-6, 2020.

MADUREIRA, A. M. A. S. Doenças emergentes e reemergentes na saúde coletiva [Apostilha do curso Técnico em Agente Comunitário de Saúde]. Montes Claros: IFNMG, 2015.

MATTE, D. L., CACAU, L., REIS, L. F. F. et al. Recomendações sobre o uso de equipamentos de proteção individual (EPIs) no ambiente hospitalar e prevenção de transmissão cruzada na COVID-19. ASSOBRAFIR Ciência, v.11, n.1), p.47-64, 2020.

MELO, P. R., AFONSO, A., ALMEIDA, A. B. et al. Recomendações da OMD para a retomada da atividade em medicina dentária durante a fase de mitigação da pandemia COVID-19, 2020. https://www.uc.pt/fmuc/MDentaria/OMD_Covid19_Recomendacoes_Junho\#: :text=\%5B13\%5D \%20Page\%208\%208\%20RECOMENDA\%C3\%87\%C3\%95ES,estendendo\%2Dse\%20at\%C3\%A $9 \% 2014 \% 20$ dias.

MONTALLI, V. A. M.; GARCEZ, A. S.; MONTALLI, G. A. M. et al. Barreira de biossegurança individual em odontologia: uma alternativa nos tempos de Covid-19: estudo preliminar. Revista Gaúcha de Odontologia, v.68, p.1-11, 2020.

MORAIS, H. G. DE F., GALVÃO, M. H. R., SILVA, W. R. DA et al. Conhecimentos, atitudes e práticas de biossegurança por cirurgiões-dentistas brasileiros durante a pandemia da COVID-19. Research, Society and Development, v.9, n.10, p.1-11, 2020.

NOGUEIRA, S. A.; BASTOS, L. F.; COSTA, I. C. C. Riscos Ocupacionais em Odontologia: Revisão da Literatura. UNOPAR Cientifica. Ciências biológicas e da saúde, v. 12, n.3, p.1120, 2010.

PERES, D.; BOLÉO-TOME, J. P.; SANTOS, G. Proteção facial e respiratória: Perspectivas atuais no contexto da pandemia por COVID-19. Acta Médica Portuguesa, v.33, n.9, p.583-592, 2020.

SILVA, A. B.; TOMAZ, L. S.; BARJA, P. R. et al. Proteção respiratória: indicações de uso durante a pandemia de COVID-19. Brazilian Journal of Development, v.7, n.3, p.30736-30752, 2021.

SILVEIRA, M. G. S. S.; FERNANDEZ, M. S.; TILLMANN, T. F. F. et al. Mudanças na prática odontológica em tempos de COVID-19: revisão e recomendações para o cuidado odontológico. Revista Gaúcha de Odontologia, v.69, p.1-10, 2021.

SOARES, S. S. S.; SOUZA, N. V. D. O.; SILVA, K. G. et al. Pandemia de Covid-19 e o uso racional de equipamentos de proteção individual. Revista Enfermagem UERJ, v.28, p.1-6, 2020.

SOUZA, A. A.; SILVA, J. C. F.; LOUREIRO, B. B. et al. Impacto da pandemia de COVID-19 em cirurgiões-dentistas no Brasil no ano de 2020: um estudo epidemiológico. Revista de Odontologia da UNESP, v.50, n.1, p.1-9, 2021. 